\title{
Linkage Analysis in Dictyostelium discoideum using Multiply Marked Tester Strains: Establishment of Linkage Group VII and the Reassessment of Earlier Linkage Data
}

\author{
By DAVID I. RATNER* AND PETER C. NEWELL \\ Department of Biochemistry, University of Oxford, South Parks Road, Oxford OX1 $3 Q U$
}

(Received 12 May 1978)

\begin{abstract}
To aid linkage analysis and mapping studies in Dictyostelium discoideum, we have constructed several tester strains with easily scored mutations characterizing the six currently identified linkage groups. Use has been made of conditionally lethal mutants unable to grow upon Bacillus subtilis, and the locus of the mutation involved $(b s g A)$ has been assigned to linkage group III. The mutation cobAl, which confers resistance to cobaltous chloride, has been assigned to a previously unidentified linkage group (VII). The temperature-sensitive growth mutation $t s g C 7$, previously reported to define linkage group $\mathrm{V}$, has been reassigned to group III, leaving linkage group V presently unmarked. The further use of genetic tester strains is described.
\end{abstract}

\section{INTRODUCTION}

Genetic studies of the cellular slime mould Dictyostelium discoideum are based upon a parasexual cycle. Haploid amoebae spontaneously fuse, at low frequency, to form diploid cells; these, in turn, occasionally revert to the haploid state (Sussman \& Sussman, 1962). Both haploid and diploid strains are sufficiently stable for their properties to be analysed; yet the interconversion of these two states is readily achieved in the laboratory (Loomis, 1969 ; Katz \& Sussman, 1972). The transition from diploidy to haploidy is believed to occur via a transient aneuploid phase (Sinha \& Ashworth, 1969; Brody \& Williams, 1974) during which intact chromosomes are lost at random during successive mitoses. Thus, when a heterozygous diploid strain generates haploid progeny, mutations on any one chromosome remain linked, whereas there is free assortment of markers on separate chromosomes (Katz \& Sussman, 1972; Williams et al., 1974).

A variety of cytological examinations [originally that of Wilson (1952), most convincingly those of Robson \& Williams (1977) and Zada-Hames (1977)] has revealed the haploid chromosome number of $D$. discoideum to be seven; consequently, seven genetically defined linkage groups are expected. To date, roughly 50 loci have been assigned to six linkage groups (reviewed in Newell, 1978), but only four of these groups are extensively marked. Therefore, at least one additional linkage group is anticipated, and the data defining some of the known groups are in need of confirmation.

Recently, parasexual manipulations of $D$. discoideum have been facilitated by the introduction of a new method for the isolation of heterozygous diploids (Newell et al., 1977a). This approach involves the fusion of a haploid strain bearing a recessive mutation for the inability to grow on the bacterium Bacillus subtilis (locus $b s g A$ ) with a second haploid strain bearing a mutation for temperature-sensitive growth ( $t s g$ ). Diploid strains formed by the

* Present address: Department of Cellular Biology, Scripps Clinic and Research Foundation, 10666 North Torrey Pines Road, La Jolla, California 92037, U.S.A. 
union of two such amoebae exhibit the wild-type phenotype and are readily selected by requiring growth on a $B$. subtilis lawn at the non-permissive temperature. This Bsg/Tsg fusion method offers a major advantage over the previously employed procedure for diploid selection (Loomis, 1969), in which strains carrying two complementing (i.e. non-allelic) $t s g$ mutations are fused. In the double Tsg procedure, Tsg haploid segregants subsequently derived from the diploid are of uncertain genotype; they could carry either one or both of the parental tsg mutations, and the resolution can be burdensome. In contrast, with the Bsg/Tsg method, the genotype of every haploid segregant is immediately clear from its phenotype, since only one mutation of each kind is present in the diploid. This technical advantage becomes critical when complex, multi-step strain constructions are required.

We report here the assignment of the $b s g A$ locus (on linkage group III) and the use of Bsg strains to assign other mutations to linkage groups. In particular, a mutation (termed $c o b$; Williams \& Newell, 1976) providing resistance to cobaltous chloride defines a new linkage group, group VII. Furthermore, we have demonstrated that the temperature sensitivity locus $t s g C$, previously the sole marker reported to map on linkage group V (Williams et al., 1974; Kessin et al., 1974) is actually located on group III. Based on these findings, we have constructed several well-marked genetic tester strains that should facilitate the mapping of new mutations.

\section{METHODS}

Media and chemicals. The composition and methods of preparation of nutrient (SM) and selective media, and the chemicals used, were as described previously (Mosses et al., 1975) except that Davis agar (Davis Gelatine, Christchurch, New Zealand; 'standard ' grade) was used in nutrient plates and in those containing methanol $(2 \%, \mathrm{v} / \mathrm{v})$ or cycloheximide $\left(500 \mu \mathrm{g} \mathrm{ml}^{-1}\right)$. Selective plates containing $\mathrm{CoCl}_{2}, 6 \mathrm{H}_{2} \mathrm{O}\left(300 \mu \mathrm{g} \mathrm{ml}^{-1}\right)$ were made as described by Williams \& Newell (1976), except that Difco Bacto-agar was used. The inhibitory effect of $\mathrm{CoCl}_{2}$ on bacteria and amoebae is influenced by several factors including impurities in the water and in the brand of agar used; workers in other laboratories may have to adjust the inhibitor concentration to suit their own conditions. Dextran (clinical grade) was obtained from Sigma and $p$-nitrophenyl- $\alpha$-Dmannopyranoside (A grade) from Calbiochem.

Strains. Dictyostelium discoideum stocks were grown on lawns of Klebsiella aerogenes. A cobalt-resistant mutant of $K$. aerogenes strain 1033 [isolated by Williams \& Newell (1976) and designated $K$. aerogenes strain oxf1] was used throughout. The Bacillus subtilis strain 36.1 , which was used for the selection of $D$. discoideum diploids, has been described (Newell et al., 1977a).

The $D$. discoideum strains used are derivatives of the NC4 isolate of Raper (1935). A nomenclature system based on that of Demerec et al. (1966) has been employed (Kessin et al., 1974). The origins and genotypes of haploid strains are given in Table 1. Phenotypes, when given in subsequent tables and in the text, are denoted by the mutant symbol with an initial capital (in roman type), the wild type being designated by the superscript + . Hence, Cyc describes a mutant (at the $c y c A$ locus) resistant to cycloheximide and $\mathrm{Cyc}^{+}$ describes any wild-type strain sensitive to the drug. Diploid strains of $D$. discoideum have not been tabulated; instead, their haploid parents are noted and described fully.

Maintenance of stocks. Standard procedures (Williams et al., 1974) were employed for the handling of amoebae and the preservation of spores in silica gel. Additionally, strains were preserved for intermediate periods by adding $0.2 \mathrm{ml}$ of spores suspended in horse serum (as prepared for silica gel storage) to $5 \mathrm{ml}$ of sterile aqueous $80 \%(\mathrm{v} / \mathrm{v})$ glycerol; kept at $-20^{\circ} \mathrm{C}$, the suspension remained viable for several months at least.

Genetic manipulations. Diploid strains were formed using the Bsg/Tsg fusion system of Newell et al. (1977a, b). In this method, amoebae of one Bsg haploid and one Tsg haploid strain were suspended together overnight and then plated on to lawns of $B$. subtilis incubated at $27^{\circ} \mathrm{C}$. Diploids were recovered at a frequency of $10^{-5}$. Diploids formed from complementing Tsg mutants (Loomis, 1969) were also isolated on B. subtilis lawns (Newell et al., 1977a). Presumptive diploid clones, appearing on the restrictive plates in 4 or $5 \mathrm{~d}$, were recloned on $K$. aerogenes lawns at $22{ }^{\circ} \mathrm{C}$ prior to genetic testing and confirmation of ploidy. In many experiments, we retained several of the diploid clones appearing on the selective plates for further study: these strains are likely to result from independent fusion events.

Haploid segregants were isolated from heterozygous diploids by standard methods (see, for example, Williams et al., 1974) using cycloheximide, methanol, or $\mathrm{CoCl}_{2}$ as the selective agent. In most experiments, amoebae from several freshly recloned diploid colonies were applied, in parallel, to the selective plates; the use of recently recloned amoebae ( 4 to $6 \mathrm{~d}$-old clones), and the analysis of haploids from more than one 


\section{Table 1. Strains of Dictyostelium discoideum}

Genotypic symbols are standard and have been described elsewhere (Newell et al., 1977b). The mutant phenotypes corresponding to each genotypic symbol are: $c y c A$, resistance to cycloheximide; $\operatorname{spr} A$, round spores; whi $A$, white spore mass; acr $A$, resistance to acriflavin and methanol; axe $A$, axe $B$, axenic growth (both mutations necessary); $t s g$, temperature $\left(27^{\circ} \mathrm{C}\right)$ sensitive growth; $b s g A$, inability to grow on $B$. subtilis; $b w n A$, presence of brown pigment during development; $e b r$, resistance to ethidium bromide; $\operatorname{man} A$, deficiency in $\alpha$-mannosidase- 1 ; $\operatorname{cob} A$, resistance to cobalt. Mutations are noted by their allele number; no notation is entered for wild-type loci.

Strains denoted by the letters NP or Ts were obtained from a haploid parent by screening or drug selection. Strains denoted X, XM or XP are haploid segregants of a diploid strain which was itself formed by the fusion of the two haploid strains listed as parents. Only haploid strains are shown in the Table. Strain NP167 was isolated on ethidium bromide agar as described by Wright et al. (1977); strain NP354 was isolated on methanol agar as described by Mosses et al. (1975). The mutation in NP354 that confers methanol resistance was confirmed to be at the acrA locus by complementation analysis (D. I. Ratner, unpublished results, following the method of Williams et al., 1974). Strain HU77 was isolated by K. L. Williams from a methanol-resistant diploid strain (DU220) in which a mitotic cross-over event had occurred on linkage group II, generating the nonparental genotype shown. This affords the useful combination of markers whiA, acrA, $t s g D^{+}$.

Tabulated genotypes are believed to be complete, although the presence of a single axenic allele is difficult to ascertain. The axenic loci in NP7 (and its derivative NP354) are questionable (see Discussion) but are given as in the original description of that strain.

\begin{tabular}{|c|c|c|c|c|c|c|c|c|c|c|c|c|}
\hline \multirow[b]{2}{*}{ Strain } & \multirow[b]{2}{*}{ Parent(s) } & \multicolumn{10}{|c|}{ Genotype } & \multirow{2}{*}{$\begin{array}{r}\text { Refer- } \\
\text { ence or } \\
\text { source* }^{*}\end{array}$} \\
\hline & & $c y c A$ & $\operatorname{spr} A$ & whiA & $a c r A$ & axe & tsg & $b s g A$ & $b w n A e b r$ & $\operatorname{man} A$ & $\operatorname{cob} A$ & \\
\hline Ts12 & $\mathrm{NC} 4$ & 1 & & 1 & & & $D I 2$ & & & & & 1,2 \\
\hline NP7 & $\mathrm{A} \times 3$ & & & & & $A 1, B 1$ & $C 7$ & & & & & 3 \\
\hline NP12 & NP3 & & & & 2 & $A 1, B 1$ & $B 3$ & & & & & 2 \\
\hline NP167 & NP12 & & & & 2 & $A 1, B 1$ & $B 3$ & & 404 & & & 4 \\
\hline NP194 & NP20 & 5 & & & & & & 5 & & & & 5 \\
\hline NP354 & NP7 & & & & 400 & $A 1, B 1$ & $C 7$ & & & & & 6 \\
\hline HU77 & Ts12, NP12 & 1 & & $I$ & 2 & $A 1, B 1 ?$ & $B 3$ & & & & & 4 \\
\hline XM101 & $\mathrm{x} 9, \mathrm{M} 2-88$ & 1 & & 1 & 1 & & $D 12$ & & 1 & 2 & & 7 \\
\hline & NP2, M28 & & & & & $A 1, B 1$ & $A 1$ & & 1 & & & 2 \\
\hline$\times 22$ & NP14, M28 & & 1 & 1 & 1 & & $D 12, E 13$ & & & & & 8 \\
\hline$x 36$ & NP62, NP68 & 1 & & & & $A 1, B 1$ & $A I$ & & 1 & & $I$ & 8 \\
\hline XP55 & NP194, X22 & 5 & & & & & & 5 & & & & 5 \\
\hline XP58 & x36, XP55 & 1 or 5 & & & & & & 5 & & & 1 & 6 \\
\hline XP64 & XP58, XM101 & 1 or 5 & & & & & & 5 & & 2 & 1 & 6 \\
\hline XP65 & XP58, XM101 & 1 or 5 & & & & & & 5 & & 2 & 1 & 6 \\
\hline XP71 & $\mathrm{x} 2, \mathrm{HU} 77$ & & & $I$ & 2 & $A 1, B I$ & $A I$ & & 1 & & & 6 \\
\hline XP72 & $x 2$, HU 77 & & & 1 & 2 & $A 1, B 1$ & $A I$ & & $l$ & & & 6 \\
\hline XP92 & XP65, XP72 & 1 or 5 & & & & BI & $A 1$ & & & 2 & 1 & 6 \\
\hline XP95 & XP64, XP71 & & & 1 & 2 & $A 1, B 1$ & $A I$ & & 1 & 2 & 1 & 6 \\
\hline XP99 & XP64, XP71 & 1 or 5 & & & & & & 5 & 1 & 2 & 1 & 6 \\
\hline
\end{tabular}

* 1, Katz \& Sussman (1972); 2, Williams et al. (1974); 3, Kessin et al. (1974); 4, K. L. Williams, unpublished; 5, Newell et al. (1977a); 6, this study; 7, Free et al. (1976); 8, Williams \& Newell (1976).

clone, seem to reduce unwanted bias in the segregant population (Welker \& Deering, 1978; Newell et al., $1977 b$ ). Nevertheless, it was helpful to vary the number of amoebae per selective plate to ensure that more slowly growing segregants (for example, those containing the $t s g C 7$ mutation) could be recovered without

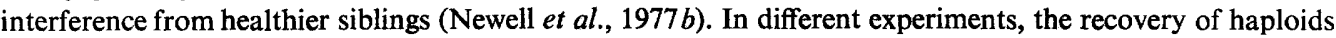
ranged from $1 \times 10^{-4}$ to $3 \times 10^{-3}$. The recovery of interfering, drug-resistant diploids (arising by mitotic recombination) varied depending upon the diploid parent and the selective agent; diploid segregants were detected by spore shape and eliminated from the linkage analyses.

Artifactual appearance of $\mathrm{Tsg}^{+} \mathrm{Bsg}^{+}$segregants. A methodological point which has not been commented upon previously is the need for recloning haploid segregants displaying certain phenotypes. For example, seeming $\mathrm{Tsg}^{+} \mathrm{Bsg}^{+}$haploid segregant colonies have occasionally been shown, upon further clonal purification, to consist of a mixture only of Tsg Bsg ${ }^{+}$and $\mathrm{Tsg}^{+} \mathrm{Bsg}$ amoebae or, in some cases, to contain small numbers of parental diploids. The initial mixed colonies presumably resulted from either contamination with the parental diploid (which is $\mathbf{B s g}^{+} \mathbf{T s g}^{+}$) or haploidization on the selective plate of an amoeba which was aneuploid at the time of plating. Whatever their origin, failure to reclone and then retest such segregants 
can lead to incorrect interpretation of linkage data. [For example, $b s g A$ failed to show linkage to $\operatorname{tsg} A$ on group III in the initial experiments of Newell et al. $(1977 \mathrm{~b})$.] This is not a general problem with most haploid marker tests as these are either non-selective (e.g. colour markers) or select against the parental diploids (e.g. drug markers).

Determination of segregant phenotypes. The scoring of most phenotypes followed standard methods (Williams et al., 1974). We note only the following modifications. Growth on plates containing $\mathrm{CoCl}_{2}$ was scored somewhat later than on other drug plates (typically 7 to $9 \mathrm{~d}$ after testing). At times we found it useful to pre-incubate (overnight at $27^{\circ} \mathrm{C}$ ) the $\mathrm{K}$. aerogenes lawn on $\mathrm{CoCl}_{2}$-containing plates: this increased bacterial growth and thereby made the amoebal tests more uniform.

Discrimination between Bsg and Bsg ${ }^{+}$haploids was obvious 6 to $8 \mathrm{~d}$ (at $22{ }^{\circ} \mathrm{C}$ ) after transferring amoebae (using toothpicks) on to an agar plate spread with $0.1 \mathrm{ml}$ of $B$. subtilis freshly suspended in sterile dihydrostreptomycin sulphate $\left(2 \mathrm{mg} \mathrm{m}^{-1}\right)$. At earlier times (4 to $5 \mathrm{~d}$ ), Bsg strains often showed slight growth (but sometimes comparable to that of $\mathbf{B s g}^{+}$controls) apparently due to the utilization of residual $K$. aerogenes applied together with, and possibly pre-ingested by, the amoebae. Transfer of spore masses rather than amoebae made the phenotypic distinction even more striking.

Scoring of the Man ( $\alpha$-mannosidase-1) phenotype was accomplished by a simplification of the procedure of Free et al. (1976). Developing amoebae (about $10^{6}$ cells, taken with a sterile loop from slugs formed on the SM agar test plates) were added to $0.5 \mathrm{ml}$ portions of the complete assay mixture (0.01 M-p-nitrophenyl$\alpha$-D-mannopyranoside freshly mixed with an equal volume of $5 \mathrm{mM}$-cysteine $/ 0 \cdot 1 \mathrm{M}$-acetate buffer, $\mathrm{pH} 5 \cdot 0$ ) pre-distributed into 24-well plastic grids (Linbro). After incubation at $35^{\circ} \mathrm{C}$ for $60 \mathrm{~min}$, the addition of $0.5 \mathrm{ml}$ of $1 \mathrm{M}-\mathrm{Na}_{2} \mathrm{CO}_{3}$ revealed the presence (suspension turned bright yellow) or absence (suspension remained colourless) of the enzyme. Controls indicated that the deliberate lysis of the amoebae (Free et al., 1976), either by freezing and thawing or by adding detergent, could be omitted without hindering the assay.

\section{RESULTS}

\section{Assignment of the bsgA locus to linkage group III}

Our initial report of the B. subtilis sensitivity mutation (Newell et al., 1977a) included data showing the independent segregation of the mutation from the well-marked linkage groups I and II. To test the relationship of the bsg locus to groups III and IV, diploid strains DP873 and DP874 were constructed by the parasexual fusion of a $B$. subtilis-sensitive strain (NP194) with a partner (x2) carrying the markers $t$ sg $A$ and bwnA. (The complete genotypes of haploid strains appear in Table 1.) By plating diploid cultures on to selective agar plates containing cycloheximide, we recovered haploid segregants whose phenotypes are shown in Table 2. The presence of clones of a recombinant (i.e. non-parental) phenotype with respect to their bsg and $c y c A$ alleles confirmed that the bsg locus is not on linkage group I. The existence of recombinant segregants of $b s g$ and $b w n A$ similarly established that $b s g$ is not on group IV. In contrast, only parental-type haploids were recovered when bsg was tabulated against $t s g A$. Thus the bsg mutation resides on linkage group III, and the locus is henceforth designated $b s g A$. The observation of a deviation from randomness (roughly a fourfold bias of Bsg Tsg ${ }^{+}$to $\mathrm{Bsg}^{+}$Tsg segregants) has been commented upon by several authors (Rothman \& Alexander, 1975; Wright et al., 1977; Newell et al., 1977b). In this case, it is presumed to reflect the presence of a deleterious mutation on linkage group III of strain $\mathbf{x} 2$.

\section{Assignment of the cobA locus to linkage group VII}

Williams \& Newell (1976) isolated a mutation, cob-1, that provides resistance to cobalt ions. The mutation appeared to be an important genetic marker because of indications that it was not on any of the linkage groups known at that time, although no linkage data were presented. The cob-1 mutation has subsequently been used in other genetic studies (Coukell \& Roxby, 1977; Welker \& Deering, 1978), but its linkage group has not been determined. The simplicity of the Bsg/Tsg fusion system enabled us to determine the linkage group of the cob- 1 mutation unambiguously.

An early experiment to this end involved the segregation of haploid derivatives from a diploid strain (DP762) formed by the fusion of strains XP58 (cycA;bsgA;cob-1) and XM101 


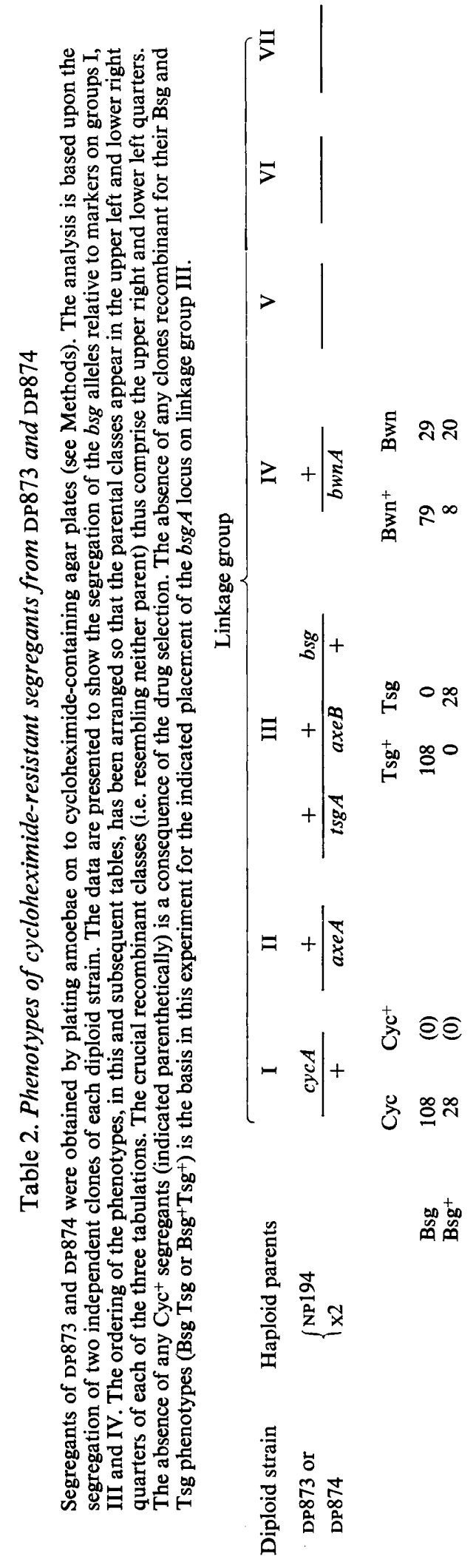




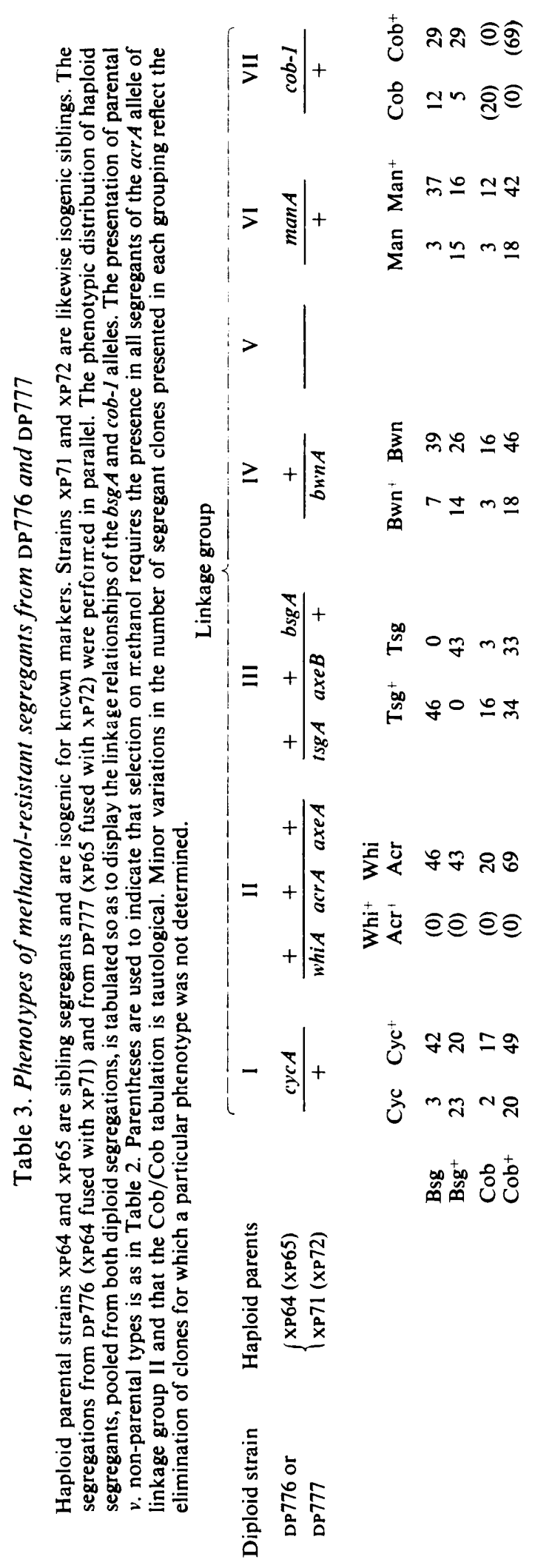


(cycA; whiA, acrA, tsg $D ; b w n A ; \operatorname{man} A)$. Among the haploid segregants were those of the genotype represented by strain XP64 $(c y c A ; b s g A ; \operatorname{man} A ; c o b-1)$. The existence of these and of other non-parental segregants not detailed here again suggested (cf. Williams \& Newell, 1976) that the $c o b-1$ mutation does not reside on linkage groups I to IV, and further indicated that the mutation is not on group VI, defined by the manA locus.

To establish this lack of linkage with rigour, we fused strain XP64 to strain XP71, containing markers for several other linkage groups. The resulting diploid, DP776, was analysed by the segregation of haploids on methanol-containing plates, with the results displayed in Table 3. The pattern of phenotypes recovered confirmed the placement of $b s g A$ on linkage group III, in repulsion to $t s g A$. On the other hand, $c o b-1$ segregated freely from each of the five linkage groups (I, II, III, IV and VI) tested simultaneously in this experiment. The same conclusions were also derived from subsequent experiments in which haploids were obtained from DP776 (or DP777, cf. Table 3) by selection on plates containing cycloheximide or $\mathrm{CoCl}_{2}$ rather than methanol (data not shown).

To test the possible linkage of $c o b-1$ to the remaining linkage group, group V, strain XP64 was fused with strain NP7, in which the $t s g C$ mutation defined that group. The diploid formed, DP768, was analysed by means of cycloheximide selection, with the results shown in Table 4. Once again, $c o b-1$ segregated freely with respect to linkage groups $\mathrm{I}(c y c A)$, III $(b s g A)$ and VI (man $A)$. In addition the results showed the absence of linkage of $c o b-I$ to $t s g C$. We have also examined two other diploids, of certain independent origin but isogenic with DP768 (strains DP769 and DP770), the segregation patterns of which confirm the lack of linkage between $c o b-1$ and groups I, III, V and VI (data not shown). The conclusion follows that the $c o b-1$ mutation is on none of the known linkage groups I to VI and therefore specifies a new genetic linkage group, group VII. The locus is designated $\operatorname{cob} A$.

\section{A critical re-examination of linkage group $V$}

The data in Table 4 are disturbing, however, in that the $b s g A$ locus mapped in opposition to $t s g C$ on linkage group $\mathrm{V}$. This result would seem to contradict our previous placement of $b s g A$ on group III. Moreover, the contradiction is not peculiar to this experiment: the linkage of $b s g A$ to $t \operatorname{sg} C$ has been observed in a variety of independent segregations (using either cycloheximide or $\mathrm{CoCl}_{2}$ selection) from DP768, DP769 and DP770. In all, from 10 separate haploid selections, 330 parental-type segregants were recovered (277 $\mathrm{Bsg} \mathrm{Tsg}^{+}$; $53 \mathrm{Bsg}^{+}$Tsg) but not a single recombinant haploid. Additionally, we have studied the segregation of these markers using the original Bsg isolate, NP194, and also a strain derived directly from NP194, strain XP55. In all cases, $b s g A$ opposed the $t s g C$ allele on linkage group $\mathrm{V}$ (97 parental segregants, 0 recombinants; data from four independent drug selections).

Our previous assertion, namely that $b s g A$ is on linkage group III, is supported by much additional evidence. An experiment analogous to that presented in Table 2 was performed by the segregation on $\mathrm{CoCl}_{2}$ plates of a diploid (DP748) constructed by the fusion of XP55 $(c y c A ; b s g A)$ with $\times 36(c y c A ; \operatorname{axe} A ; \operatorname{tsg} A, \operatorname{axe} B ; b w n A ; \operatorname{cob} A)$. The 80 clones examined consisted entirely of $\mathrm{Bsg} \mathrm{Tsg}^{+}$and $\mathrm{Bsg}^{+} \mathrm{Tsg}$ parental types, indicating the repulsion of $b s g A$ and $\operatorname{tsg} A$ on linkage group III. Furthermore, we have used the axeB locus to mark group III, in order to provide a test that is independent of the $\operatorname{tsg} A$ locus. For this experiment, diploids were constructed from strains XP55 (cycA; bsg $A$ ) and NP167 (acr A, axe $A$; axe B; $t s g B ; e b r-404$ ), and then segregated on methanol to force the presence of axe $A$ (linked to $\operatorname{acr} A$ ) in all haploid segregants. Behaviour in axenic medium thus reflected the segregation of the other axenic gene, axe $B$ (since axe $A$ alone is insufficient to permit axenic growth; Williams et al., 1974). Fifty-eight haploids recovered from two independent diploids consisted of $37 \mathrm{Bsg} \mathrm{Axe}^{+}$and $21 \mathrm{Bsg}^{+}$Axe parental types. No haploids recombinant for the $b s g A$ and $a x e B$ loci were observed. Thus, once again, $b s g A$ mapped to group III.

Because of the apparent linkage of $b s g A$ to groups III and V, we conducted an independent test of the linkage relations of $t s g C$. Strain NP354 (acrA, axeA; axeB; tsgC) was fused with 


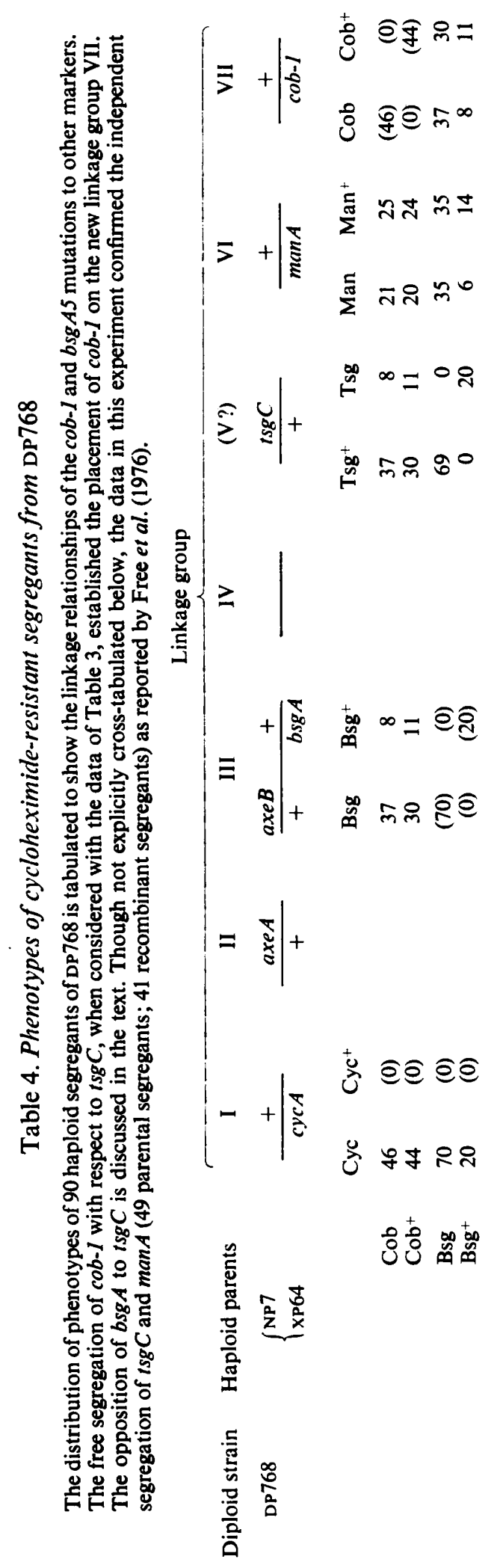




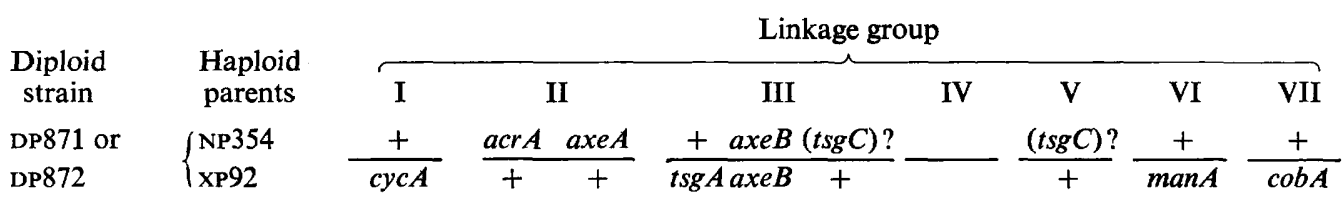

Fig. 1. Genotype of strains DP871 and DP872, showing the two placements considered for the $t s g C$ locus. The recovery, upon methanol selection, of only temperature-sensitive segregants proved that the correct assignment of $t s g C$ is to linkage group III.

a multiply marked tester strain XP92 (cycA;tsgA, axeB; man $A ; \operatorname{cob} A)$. The resulting diploids (DP871 and DP872; Fig. 1) were then plated on to methanol plates and segregants were examined from eight independent methanol selections. If $t s g C$ and $t s g A$ were on different linkage groups as previously reported (Williams et al., 1974), one-quarter or more of the segregants would be expected to be temperature-resistant. (A priori, $25 \%$ of the haploids would receive both the $t s g A^{+}$and $t s g C^{+}$alleles; but in practice we often observed a bias in favour of $t s g A^{+}$over $t s g A$, and $t s g C^{+}$over $t s g C$, as evident in Tables 2 and 4 . Hence we would predict at least $25 \%$ temperature-resistant segregants in this experiment.) If, on the other hand, $t s g C$ were actually on linkage group III in opposition to $t s g A$, then every haploid segregant must contain one or other of the temperature-sensitive alleles. We observed 190 temperature-sensitive haploid segregants but not a single temperature-resistant one. This result argues strongly that $t s g C$ opposes $t s g A$ on group III. [The alternative interpretation, in which $t s g C$ would be linked to the selected $a c r A$ allele, is incompatible with numerous other experiments (Kessin et al., 1974; Free et al., 1976; D. I. Ratner, unpublished observations) showing free segregation between $t s g C$ and linkage group II.] That these eight independent selections did not suffer from any unanticipated bias was indicated by the random distribution of the unselected markers $c y c A, \operatorname{man} A$ and $\operatorname{cob} A(42,48$ and $46 \%$ recovery of the respective mutant alleles). Finally, complementation tests were performed on a small subset of these segregants in which it was established that the $\operatorname{sg} A$ allele, and therefore the critical linkage group III, also segregated well: half $(11 / 20)$ of the clones tested fused with the $\operatorname{tsg} A$ parent to yield temperature-resistant diploids. In view of all these controls, we conclude that $t s g C$ does, in fact, map on linkage group III.

\section{DISCUSSION}

The $b s g A 5$ mutation was isolated in the hope that it would facilitate parasexual mapping in $D$. discoideum. This study illustrates some of the ways in which we have taken advantage of the Bsg/Tsg fusion system. A necessary beginning was to map the $b s g A$ locus. The experiments reported here place $b s g A$ on linkage group III, in opposition to each of the two loci, axeB and $t s g A$, originally used to define that group (Williams et al., 1974). The Bsg/Tsg fusion method was instrumental in proving that the $c o b-1$ mutation of Williams \& Newell (1976) segregates freely from all known linkage groups; that is, that the $\operatorname{cob} A$ locus identifies a new linkage group, VII. These same experiments, however, produced the surprising result that the $b s g A$ locus is linked to $t s g C$, which had been reported to be on group V (Williams et al., 1974; Kessin et al., 1974). The possible interpretations of this unexpected result deserve further comment.

Naively, one might imagine that the Bsg phenotype could appear to map on both groups III and V if it were the result of a double mutation, with one lesion on each of the two linkage groups. However, further reflection suggests that this model is inappropriate: if the Bsg phenotype were due to two mutations, it would segregate independently (with one class of segregant lacking) of both linkage groups, rather than being linked to both. Similarly, the TsgC phenotype cannot depend upon mutations on two linkage groups. A different explana- 


\begin{tabular}{|c|c|c|c|c|c|c|c|c|}
\hline & & & & Lin & ge grou & & & \\
\hline & & I & II & III & IV & V & VI & VII \\
\hline Parent & XP55 & $c y c A$ & & bsg $A$ & & & & \\
\hline Tester & XP95 & & $\overline{\text { whiA acrA }}$ & $\overline{t s g A}$ & $\overline{b w n A}$ & & $\overline{\operatorname{man} A}$ & $\overrightarrow{c o b A}$ \\
\hline Parent & $\times 22$ & & whiA acrA & & & & & \\
\hline Tester & XP99 & $\overline{c y c A}$ & & $\overline{b s g A}$ & $\overline{b w n A}$ & & $\overline{\operatorname{man} A}$ & $\overline{a b A}$ \\
\hline
\end{tabular}

Fig. 2. Tester strains used for mapping new mutations introduced into two standard parents, XP55 and $\mathrm{x} 22$. The mutant loci shown are those which most conveniently identify each of the known linkage groups. Tester XP95 also carries $a x e A$ and $a x e B$ mutations. The complete genotype of $\times 22$ is (sprA, tsgE; whi A, acrA, tsg D) (Williams \& Newell, 1976).

tion of the $b s g A$ data invokes a reciprocal translocation, in which some of the DNA of two chromosomes of the Bsg strain became interchanged. Such translocations are known in a variety of other organisms and do produce mitotic mapping data which can be mistaken for linkage (see, for example, Käfer, 1962). However, we have confirmed the linkage of $b s g A 5$ to both $t s g C$ and $t s g A$ in a genealogical succession of Bsg strains, without any indication of the sudden appearance of a translocation at any point in our strain construction (D. I. Ratner, unpublished observations). Finally, our demonstration (see Results) of the opposition of the $t s g C$ and $t \operatorname{sg} A$ loci, in an experiment not involving Bsg strains, confirmed an alternative explanation of the $b s g A$ data, that is, the $t s g C$ locus maps together with $b s g A$ and $t \operatorname{sg} A$ on linkage group III, rather than group $\mathrm{V}$ as originally reported.

On what basis was $t s g C$ originally asserted to define a linkage group distinct from group III ? Williams et al. (1974) reported that linkage group V, as defined by $t s g C$, could be substituted from a non-axenic strain into an axenic one without loss of axenicity (and recent experiments confirmed this observation; D. I. Ratner, unpublished observations). The experiment involved the recovery of temperature-resistant axenic segregants from a diploid formed by the fusion of NP7 with a non-axenic partner. This result would seem to suggest, as the authors concluded, that the $t s g C$ allele of $\mathrm{NP} 7$ was not linked to its axe $B$ allele. Although the parental axenic strain, Ax3, was shown to possess both axe $A$ and axe $B$ genes, this was not proved, however, for the axenic strain NP7. In fact, the axenic growth properties of strain NP7 are quite poor, with stationary titres reported of less than $10^{6}$ (Kessin et al., 1974). This value is only $5 \%$ of the stationary titre of $\mathrm{AX} 3$ (Kessin et al., 1974). It is also significant that the isolation of the $t s g C$ mutation in strain NP7 involved a period of selection in axenic medium after mutagenesis (Kessin et al., 1974). Conceivably, the limited axenic growth of strain NP7 is not the result of the functional presence of axe $B$; rather, it may reflect the presence of axe $A$ together with other mutations giving partial ability to grow axenically. From these collective observations, it would appear that the axenic mutations in strain NP7 are not suitable for linkage determinations and that the original interpretation that $t s g C$ segregated freely of linkage group III is unsubstantiated. K. L. Williams (personal communication) has recently independently confirmed the opposition of the $t \operatorname{sg} C$ and $t \operatorname{sg} A$ loci on linkage group III, in agreement with the results reported here.

Consequently, mutations now determine six genetic linkage groups in D. discoideum, out of the seven expected from cytological studies (Wilson, 1952; Robson \& Williams, 1977; Zada-Hames, 1977). Linkage group $\mathrm{V}$, previously defined only by $t s g C$, is unmarked. We specify the new linkage group of the $\operatorname{cob} A$ locus as VII (and not V) simply to conform with accepted nomenclature (Newell et al., $1977 b$; Wright et al., 1977; Welker \& Deering, 1978; Williams, 1978).

One purpose of this work was to create heavily marked 'tester' strains that will aid future mapping studies. We have constructed a number of strains that contain readily scored 
mutations on the six linkage groups, in combinations appropriate for fusion with a variety of basic parental stocks into which new mutations may be introduced. The use of two such tester strains is illustrated in Fig. 2. Strain XP95 is highly suitable for mapping new mutations in the Bsg strain XP55: XP55/XP95 diploids are heterozygous for all the markers shown, so that all known linkage groups are tested in a single genetic experiment. Strain XP55 itself grows and develops very well and is used in this laboratory as a parental strain for obtaining developmental mutants of several kinds. Analogously, tester strain XP99 is useful for mapping mutations in another common parental stock, x22 (Williams \& Newell, 1976). Testers have already been used to discover other loci on linkage group VII : mutation at one of these sites results in temperature sensitivity (tsgK; P. C. Newell, unpublished observations), whereas mutation at the other site leads to unusually large aggregation streams $(\operatorname{stm} A$; F. Ross, unpublished observations). Tester strains are very useful in mapping complex developmental phenotypes which are the result of multiple mutations (D. I. Ratner, unpublished observations). By analogy with other systems (Käfer, 1962), testers may ultimately establish the existence of chromosomal translocations in $D$. discoideum and should, in any case, aid in the search for loci that identify the last remaining linkage group.

We wish to thank Stephanie Rogers for excellent technical assistance and K. L. Williams for frequent communication of unpublished results. Strain HU77 was kindly provided by K. L. Williams and XM101 by W. F. Loomis. D.I.R. received support from the U.S. National Institutes of Health, Division of General Medical Sciences.

\section{REFERENCES}

Brody, T. \& Williams, K. L. (1974). Cytological analysis of the parasexual cycle in Dictyostelium discoideum. Journal of General Microbiology 82, 371-383.

Coukell, M. B. \& RoxBy, N. M. (1977). Linkage analysis of developmental mutations in aggregation-deficient mutants of Dictyostelium discoideum. Molecular and General Genetics 151, 275-288.

Demerec, M., Adelberg, E. A., Clark, A. J. \& HARTMAN, P. E. (1966). A proposal for a uniform nomenclature in bacterial genetics. Genetics 54, 61-76.

Free, S. J., Schimke, R. T. \& Loomis, W. F. (1976). The structural gene for $\alpha$-mannosidase-1 in Dictyostelium discoideum. Genetics 84, 159-174.

KÄFER, E. (1962). Translocations in stock strains of Aspergillus nidulans. Genetica 33, 59-68.

Katz, E. R. \& Sussman, M. (1972). Parasexual recombination in Dictyostelium discoideum: selection of stable diploid heterozygotes and stable haploid segregants. Proceedings of the National Academy of Sciences of the United States of America 69, 495-498.

Kessin, R. H., Williams, K. L. \& Newell, P. C. (1974). Linkage analysis in Dictyostelium discoideum using temperature-sensitive growth mutants selected with bromodeoxyuridine. Journal of Bacteriology 119, 776-783.

Loomis, W. F. (1969). Temperature sensitive mutants of Dictyostelium discoideum. Journal of Bacteriology 99, 65-69.

Mosses, D., Williams, K. L. \& Newell, P. C. (1975). The use of mitotic crossing-over for genetic analysis in Dictyostelium discoideum: mapping of linkage group II. Journal of General Microbiology 90, 247-259.
Newell, P. C. (1978). Genetics of the cellular slime moulds. Annual Review of Genetics 12, 69-93. Newell, P. C., Henderson, R. F., Mosses, D. \& Ratner, D. I. (1977a). Sensitivity to Bacillus subtilis: a novel system for selection of heterozygous diploids of Dictyostelium discoideum. Journal of General Microbiology 100, 207-211.

Newell, P. C., Ratner, D. I. \& Wright, M. D. $(1977 b)$. New techniques for cell fusion and linkage analysis of Dictyostelium discoideum. In Development and Differentiation in the Cellular Slime Moulds, pp. 51-61. Edited by P. Cappuccinelli \& J. Ashworth. Amsterdam: Elsevier/North Holland.

RAPER, K. B. (1935). Dictyostelium discoideum, a new species of slime mold from decaying forest leaves. Journal of Agricultural Research 50, 135147.

Robson, G. E. \& Williams, K. L. (1977). Mitotic chromosomes of the cellular slime mould Dictyostelium discoideum: karyotype based on Giemsa banding. Journal of General Microbiology 99, 191200.

Rothman, F. G. \& Alexander, E. T. (1975). Parasexual genetic analysis of the slime mould Dictyostelium discoideum A3. Genetics 80, 715731.

Sinha, U. \& Ashworth, J. M. (1969). Evidence for the existence of elements of a parasexual cycle in the cellular slime mould Dictyostelium discoideum. Proceedings of the Royal Society B173, 531-540.

Sussman, M. \& Sussman, R. R. (1962). Ploidal inheritance in Dictyostelium discoideum: stable haploid, stable diploid, and metastable strains. Journal of General Microbiology 28, 417-429. 
Welker, D. L. \& DeEring, R. A. (1978). Genetics of radiation sensitivity in the slime mould Dictyostelium discoideum. Journal of General Microbiology 109, 11-23.

Williams, K. L. (1978). Characterization of dominant resistance to cobalt chloride in Dictyostelium discoideum and its use in parasexual genetic analysis. Genetics (in the Press).

Williams, K. L. \& Newell, P. C. (1976). A genetic study of aggregation in the cellular slime mould Dictyostelium discoideum using complementation analysis. Genetics 82, 287-307.

Williams, K. L., Kessin, R. H. \& Newell, P. C. (1974). Parasexual genetics in Dictyostelium discoideum: mitotic analysis of acriflavin resist- ance and growth in axenic medium. Journal of General Microbiology 84, 59-69.

WILson, C. M. (1952). Sexuality in the Acrasiales. Proceedings of the National Academy of Sciences of the United States of America 38, 659-662.

Wright, M. D., Williams, K. L. \& Newell, P. C. (1977). Ethidium bromide resistance: a selective marker located on linkage group IV of Dictyostelium discoideum.Journal of General Microbiology $102,423-426$.

ZaDA-HAMES, I. M. (1977). Analysis of karyotype and ploidy of Dictyostelium discoideum using colchicine-induced metaphase arrest. Journal of General Microbiology 99, 201-208. 